\title{
Genetic identification and characterization of chromosomal regions for kernel length and width increase from tetraploid wheat
}

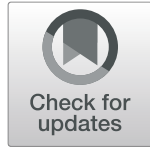

Jieguang Zhou ${ }^{1 \dagger}$, Cong $\mathrm{Li}^{1+}$, Jianing You${ }^{1}$, Huaping Tang ${ }^{1}$, Yang Mu', Qiantao Jiang ${ }^{1}$, Yaxi Liu', Guoyue Chen ${ }^{1}$, Jirui Wang ${ }^{1}$, Pengfei Qi ${ }^{1}$, Jun Ma ${ }^{2}$, Yutian Gao ${ }^{2}$, Ahsan Habib ${ }^{3}$, Yuming Wei ${ }^{1}$, Youliang Zheng ${ }^{1}$, Xiujin Lan ${ }^{1}$ and Jian $\mathrm{Ma}^{1 *}$

\begin{abstract}
Background: Improvement of wheat gercTriticum aestivum L.) yield could relieve global food shortages. Kernel size, as an important component of 1000-kernel weight (TKW), is always a significant consideration to improve yield for wheat breeders. Wheat related species possesses numerous elite genes that can be introduced into wheat breeding. It is thus vital to explore, identify, and introduce new genetic resources for kernel size from wheat wild relatives to increase wheat yield.

Results: In the present study, quantitative trait loci (QTL) for kernel length (KL) and width (KW) were detected in a recombinant inbred line (RIL) population derived from a cross between a wild emmer accession 'LM001' and a Sichuan endemic tetraploid wheat 'Ailanmai' using the Wheat $55 \mathrm{~K}$ single nucleotide polymorphism (SNP) arraybased constructed linkage map and phenotype from six different environments. We identified eleven QTL for KL and KW including two major ones QKL.sicau-AM-3B and QKW.sicau-AM-4B, the positive alleles of which were from LM001 and Ailanmai, respectively. They explained 17.57 to $44.28 \%$ and 13.91 to $39.01 \%$ of the phenotypic variance, respectively. For these two major QTL, Kompetitive allele-specific PCR (KASP) markers were developed and used to successfully validate their effects in three $F_{3}$ populations and two natural populations containing a panel of 272 Chinese wheat landraces and that of 300 Chinese wheat cultivars, respectively. QKL.sicau-AM-3B was located at 675.6-695.4 Mb on chromosome arm 3BL. QKW.sicau-AM-4B was located at 444.2-474.0 Mb on chromosome arm 4BL. Comparison with previous studies suggested that these two major QTL were likely new loci. Further analysis indicated that the positive alleles of QKL.sicau-AM-3B and QKW.sicau-AM-4B had a great additive effect increasing TKW by $6.01 \%$. Correlation analysis between $\mathrm{KL}$ and other agronomic traits showed that $\mathrm{KL}$ was significantly correlated to spike length, length of uppermost internode, TKW, and flag leaf length. KW was also significantly correlated with TKW. Four genes, TRIDC3BG062390, TRIDC3BG062400, TRIDC4BG037810, and TRIDC4BG037830, associated with kernel development were predicted in physical intervals harboring these two major QTL on wild emmer and Chinese Spring reference genomes.
\end{abstract}

\footnotetext{
* Correspondence: jianma@sicau.edu.cn

†Jieguang Zhou and Cong Li contributed equally to this work.

'State Key Laboratory of Crop Gene Exploration and Utilization in Southwest

China, Triticeae Research Institute, Sichuan Agricultural University, Chengdu

611130, China

Full list of author information is available at the end of the article
}

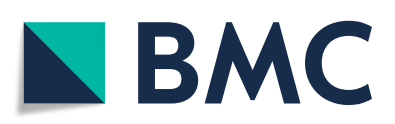

(- The Author(s). 2021 Open Access This article is licensed under a Creative Commons Attribution 4.0 International License, which permits use, sharing, adaptation, distribution and reproduction in any medium or format, as long as you give appropriate credit to the original author(s) and the source, provide a link to the Creative Commons licence, and indicate if changes were made. The images or other third party material in this article are included in the article's Creative Commons licence, unless indicated otherwise in a credit line to the material. If material is not included in the article's Creative Commons licence and your intended use is not permitted by statutory regulation or exceeds the permitted use, you will need to obtain permission directly from the copyright holder. To view a copy of this licence, visit http://creativecommons.org/licenses/by/4.0/ The Creative Commons Public Domain Dedication waiver (http://creativecommons.org/publicdomain/zero/1.0/) applies to the data made available in this article, unless otherwise stated in a credit line to the data. 


\begin{abstract}
Conclusions: Two stable and major QTL for KL and KW across six environments were detected and verified in three biparental populations and two natural populations. Significant relationships between kernel size and yield-related traits were identified. KASP markers tightly linked the two major QTL could contribute greatly to subsequent fine mapping. These results suggested the application potential of wheat related species in wheat genetic improvement.
\end{abstract}

Keywords: Tetraploid wheat, Wheat 55 K SNP array, Kernel size, QTL validation

\section{Background}

Wheat (Triticum aestivum L.) is one of the main food crops in the world [1]. The pace of population growth requires a stable increase of wheat yield [2]. Wheat yield is determined by three key components, including productive spike number per unit area, kernel number per spike, and 1000-kernel weight (TKW) [3]. TKW is mainly affected by kernel size including kernel length (KL), kernel width (KW), and kernel thickness [4]. Therefore, KL and KW play vital roles in wheat yield formation.

To date, quantitative trait loci (QTL) for kernel size have been detected on all of the wheat chromosomes [5]. For example, seven QTL for KW were detected on chromosomes 1A, 4D, 5A, 5B, 6D, and 7B [6]. Three stable QTL were identified in more than three environments, including two for KL and one for KW [7]. Xin et al. [8] identified two QTL for KW. Furthermore, several genes for kernel size have been isolated and cloned in wheat via a map-based cloning approach. For example, the grain-shape gene Tasg-D1 encoding a Ser/ Thr protein kinase glycogen synthase kinase3 was associated with formation of round grains in wheat [9]. Ketoacyl thiolase $2 B(K A T-2 B)$ involved in $\beta$-oxidation during JA synthesis played a role in determination of kernel weight [10].

Wheat breeding is facing the bottleneck of narrow genetic basis at present [11]. Fortunately, a large diversity of undeveloped genetic resources from wheat related species could contribute to meeting future wheat production challenges. It is feasible to identify and utilize novel QTL/genes for KL and KW from excellent germplasms of wheat and its related species [12]. For example, a major QTL (QGD-4BL) controlling kernel size of the upper spikelet was identified in wild emmer ( $T$. turgidum ssp. dicoccoides) [13]. Four QTL for KL and one for KW were detected in durum wheat [14]. Okamoto et al. [15] found that $P 1$ had a positive effect on $\mathrm{KL}$ in Polish wheat (T. turgidum ssp. polonicum). TtGRF4-A (ortholog of rice OsGRF4) was associated with kernel size and kernel weight in wild emmer [16].

As the progenitor of modern tetraploid and hexaploid cultivated wheat, wild emmer has the highest nucleotide diversity across the Triticum taxonomic groups making it an invaluable gene pool for the genetic improvement of wheat [17]. Thus, identification of QTL/genes for KL and KW from wild emmer will facilitate progress to meet wheat production challenges in the future. In this study, we are aiming at identifying and validating major QTL for KL and KW in a recombinant inbred line (RIL) population derived from a cross between a wild emmer accession and a Sichuan endemic tetraploid wheat 'Ailanmai'.

\section{Materials and methods Genetic populations}

Four bi-parent populations developed by the single-seed descent method were used in this study. They were derived from crosses Ailanmai $\times$ LM001 $\left(\mathrm{AM}, 121 \mathrm{~F}_{8}\right.$ RILs including parents) [18], LM001 $\times$ PI $503554\left(\mathrm{MP}, 102 \mathrm{~F}_{3}\right.$ lines), Ailanmai $\times$ AS 2268 (AAs, $102 \mathrm{~F}_{3}$ lines), and Ailanmai $\times$ PI 193877 (API, $72 \mathrm{~F}_{3}$ lines). Notably, the 121 RILs of AM were previously genotyped using the Wheat $55 \mathrm{~K}$ SNP array [18] and used for QTL mapping in this study. The other three populations were used for validating QTL identified in this study. Ailanmai (T. turgidum L. $2 n=4 x=28$, AABB) is a local dwarf variety from Sichuan province, and LM001 is a wild emmer accession ( $T$. turgidum ssp. dicoccoides, $2 n=4 x=28$, AABB). PI 503554 (T. turgidum ssp. durum) and PI 193877 (T. turgidum ssp. dicoccon) were from The U.S. National Plant Germplasm System (NPGS), and AS 2268 (T. carthlicum Nevski) was collected and preserved by Triticeae Research Institute of Sichuan Agricultural University. Besides, two natural populations were further used to verify the effect of the major QTL, and they were: (I) a panel of 272 Chinese wheat landraces (CWL) genotyped using the Wheat $660 \mathrm{~K}$ SNP array [19], and (II) a panel of 300 Chinese wheat cultivars (CWC) genotyped using the Wheat $55 \mathrm{~K} \mathrm{SNP}$ array [20]. The information of two natural populations was listed in Table S1.

\section{Phenotypic evaluation}

The phenotype of AM RIL population was measured in six different environments, including Chongzhou $\left(103^{\circ} 38^{\prime} \mathrm{E}, 30^{\circ} 32^{\prime} \mathrm{N}\right)$ in 2017, 2018, 2019, and 2020 (2017CZ, 2018CZ, 2019CZ, and 2020CZ), Wenjiang $\left(103^{\circ} 51^{\prime} \mathrm{E}, \quad 30^{\circ} 43^{\prime} \mathrm{N}\right)$ in 2020 (2020WJ), and Ya'an $\left(103^{\circ} 0^{\prime} \mathrm{E}, 2^{\circ} 58^{\prime} \mathrm{N}\right)$ in 2020 (2020YA) in China. Details 
of all the experiments planted were consistent with previous study [18]. Field management was according to local agricultural practices [21]. Thirty kernels in each line were scanned using Epson Expression 10,000 XL. $\mathrm{KL}$ and $\mathrm{KW}$ were evaluated using WinSEEDLE (Regent Instruments Canada Inc) based on the selected objects in image [21]. Then, the average values of each line in a single environment and the best linear unbiased prediction (BLUP) value estimated from average values from different environments were used for QTL detection and further analysis. The data of spike length (SL), effective tiller number (ETN), length of uppermost internode (UIL), TKW, and grain number per spike (GNS) were retrieved from our previous study [18]. The measurement of flag leaf length (FLL) and flag leaf width (FLW) was conducted about ten days after anthesis. The FLL (from leaf bottom to the tip) and FLW (on the widest part of the leaf) were measured on five selected plants (five typical plants per row for each line) from the main tiller of each plant [22]. The phenotypic average value of each trait in multiple environments was used to calculate BLUP value of each trait for further analysis. All the observations were made during the previous experiment [18], and presentation of data of kernel size were completed in the current study along with validation of identified QTL.

The $\mathrm{F}_{2}$ populations of MP, AAs, and API were grown in $2020 \mathrm{CZ}$ and the harvested $F_{3}$ seeds for each plant (line) were used for phenotype. Their experiment planted and field management were consistent with the AM population. Thirty kernels in each plant were scanned using Epson Expression 10,000 XL. KL and KW were evaluated using WinSEEDLE (Regent Instruments Canada Inc) based on the selected objects in image [21]. Then, the average values of KL and KW were used for validating major QTL identified in this study. Details of environmental information of agronomic traits measurement were listed in Table S2.

The $272 \mathrm{CWL}$ were planted in six different environments, including 2012YA, 2013-2015WJ, and 20142015CZ [19]. The average value of each accession in a single environment was used for further analysis [19].

The $300 \mathrm{CWC}$ were planted in three different environments, including Beijing in 2018 and 2019, and Baoding in 2019 [20]. One hundred and twenty seeds of each accession were planted in a single row of $2 \mathrm{~m}$ in length with $0.7 \mathrm{~m} \mathrm{spa-}$ cing between the rows in three environments [20]. The kernel-related traits were measured using the SC-A wheat grain appearance quality image analysis system developed by the Hangzhou Wanshen Detection Technology Co [20].

\section{Data analysis}

SAS 8.0 (SAS Institute, Cary, NC, USA) was used to analyze the BLUP of the agronomic traits and the broad- sense heritability $\left(\mathrm{H}^{2}\right)$ in different environments. According to the description of Smith et al. [23], the SPSS Statistic 24.0 program (IBM SPSS, Armonk, NY, USA) was used to obtain Pearson's correlation coefficients within agronomic traits based on the BLUP values, descriptive statistical analyses, and independent sample $t$ test $(P<0.05)$. Frequency distributions of $\mathrm{KL}$ and $\mathrm{KW}$ values were plotted in the Origin 9.0 software using Gaussian distribution. The individuals of the AM RILs were divided into two groups based on the genotypes of the closest markers for each of the two major QTL, and then the differences between the two groups for the corresponding traits were analyzed. Furthermore, Excel (Microsoft Corporation, Microsoft Excel 2010, USA) was used to analyze the binary linear regression analysis.

\section{QTL mapping}

The details of DNA extraction and $55 \mathrm{~K}$ SNP array analysis of AM population refer to previous work [18]. The genetic map was constructed by Mo et al. [18].

The inclusive composite interval mapping (ICIM) in IciMapping 4.1 (https://www.isbreeding.net/) was used to detect QTL, and thousand permutations test $(p<$ 0.05) was used for defining QTL logarithm (base 10) of odds scores (LOD) threshold [24]. A LOD score of 2.5 was chosen as a threshold for considering significant QTL [25]. The QTL $\times$ Environment (QE) interaction effects were analyzed using IciMapping with the preset parameter: step $=1 \mathrm{cM}, \mathrm{PIN}=0.001, \mathrm{LOD}=5.0$. In the present study, QTL identified in more than three environments and expressed more than $10 \%$ of the phenotype variance explained (PVE) were defined to be major ones, and those with less than $1 \mathrm{cM}$ apart were treated as an identical one [26]. Furthermore, QTL were named in accordance with the International rules of Genetic Nomenclature (http://wheat.pw.usda.gov/ggpages/wgc/ 98/Intro.htm). The 'sicau' represents Sichuan Agricultural University.

\section{Marker development and QTL validation}

Two SNP markers were converted to Kompetitive allelespecific PCR (KASP) markers as previously described [26]. The KASP marker, KASP-AX-111112626 (Table S3), tightly linked to $Q K L$.sicau- $A M-3 B$, was used to verify the effect of QKL.sicau-AM-3B in MP population. While KASP-AX-108974756 (Table S3) was used to validate the effect of $Q K W$.sicau-AM- $4 B$ in two populations (AAs and API) with different genetic backgrounds.

As $F_{3}$ is a segregating generation, we selected $15 F_{3}$ kernels from each line of MP, AAs, and API populations for germination and grew them in greenhouse. Leaves of 15 seedlings were all collected and mixed for DNA extraction representing $\mathrm{F}_{2}$ genotype. High-quality genomic DNA was extracted using the Plant Genonic DNA Kit 
(Tiangen Biotech, Beijing, China), and was then used to do genotyping using KASP markers. Details of the amplification reaction system and conditions were listed in Table S3. The lines were divided into two groups (Data set 1 and 2) based on the genotyping results. Data set 1 represented lines with homozygous alleles from Ailanmai or LM001, whereas Data set 2 represented lines with homozygous alleles from the other parents. Lines with heterozygous genotype were not included for analysis. Finally, we evaluated the differences in KL or KW between the two groups with the independent sample $t$ test $(P<0.05)$ to determine the effects of the major QTL.

The flanking marker $A X-111112626$ was included in CWL natural population genotyped using the Wheat 55 K SNP array and $A X-108974756$ was included in the CWC natural population genotyped using Wheat $660 \mathrm{~K}$ SNP array. According to the genotype of these two flanking markers in the CWL and CWC populations. The lines were divided into two groups: (1) lines with identical genotype as Ailanmai. (2) lines with identical genotype as LM001. The BLUP values of KL and KW data from all environments of CWL and CWC were used to analyze the differences with the independent sample $t$-test $(P<0.05)$ between the two groups.

\section{Physical intervals of the major QTL and comparison with previously reported QTL}

In order to predicate physical intervals of the major QTL identified in this study, the sequences of their flanking markers were used to blast against ( $E$-value of 1e-5) genomes sequences of the wild emmer wheat 'Zavitan' WEWseq v2 (http://202.194.139.32/blast/ blastresult.php) [27] and the International Wheat Genome Sequencing Consortium (IWGSC) Chinese Spring (CS) RefSeq v2.1 (https://urgi.versailles.inrae.fr/ download/iwgsc/IWGSC_RefSeq_Assemblies/v2.1/) [28]. The annotations and functions of genes were retrieved on UniProt (http://www.uniprot.org/). We compared physical distances by anchoring flanking marker sequences of KL and KW QTL obtained in previous studies on CS to indicate whether the currently determined QTL were novel.

Furthermore, to identify the possible regulatory genes of $\mathrm{KL}$ and $\mathrm{KW}$, the spatio-temporal expression patterns of the genes that were identified in the intervals of QKL.sicau-AM-3B and QKW.sicau-AM-4B were analyzed using the Triticeae Multi-omics Center website (https://202.194.139.32/expression/index.html). For TraesCS4B03G0584900 and TraesCS4B03G0585000, their spatio-temporal expression patterns were analyzed using Chinese Spring cv-1 Development (single) on the Triticeae Multi-omics Center website (https://202.194.13 9.32/expression/index.html).

\section{Results}

Phenotypic data analyses

LM001 showed longer and narrower kernel than Ailanmai (Fig. 1; Table 1). The values of the KL and KW in each environment showed a continuous distribution (Fig. S1a, b). The KL and KW ranged from 5.86 to 9.43 $\mathrm{mm}$ and from 2.74 to $4.16 \mathrm{~mm}$, respectively, in the AM RIL population (Table 1). The standard deviation (STD) of $\mathrm{KL}$ and $\mathrm{KW}$ ranged from 0.45 to 0.60 and from 0.11 to 0.24 , respectively. $H^{2}$ of $\mathrm{KL}$ and $\mathrm{KW}$ were 0.79 and 0.72 , respectively (Table 1 ). The result indicated that both KL and KW had high repeatability over testing environments, suggesting KL and KW were mainly controlled by genetic factors.

\section{Correlation analyses between kernel traits and other yield-related traits}

Significant and positive correlations for $\mathrm{KL}$ and $\mathrm{KW}$ were detected in most different environments $(P<0.05)$. The correlation coefficients ranged from 0.62 to 0.82 for $\mathrm{KL}$ and from 0.29 to 0.45 for KW, respectively (Table 2).

The BLUP datasets of kernel size and yield-related traits were employed to evaluate their relationships. Correlation analysis showed that significant correlations $(P$ $<0.05$ ) were observed between KL and SL, UIL, TKW, FLL ( $r=0.32$ to 0.66 ; Fig. S2a, c, d, and g). However, there were no significant correlations between $\mathrm{KL}$ and ETN, GNS, KW, FLW ( $r=-0.061$ to 0.10 ; Fig. S2b, e, f, and $\mathrm{h})$. Moreover, KW showed significant correlations $(P<0.05)$ with TKW $(r=0.31$; Fig. S2l $)$, but the other six agronomic traits (SL, ETN, UIL, GNS, FLL and FLW) were not significantly correlated to the KW ( $r=-$ 0.16 to 0.13 ; Fig. S2i, j, k, m, n, and o).

\section{QTL detection}

A total of eleven putative QTL associated with KL (six QTL) and KW (five QTL) were identified in the AM population and they were located on chromosomes $1 \mathrm{~B}$, 2A, 2B, 3B, 4B, 6A, 6B, and 7A (Table 3).

Six QTL for KL explained 4.56 to $44.28 \%$ of the PVE. QKL.sicau-AM-3B, a major and stable locus, was detected in five environments and BLUP data, and explained 17.57 to $44.28 \%$ of the PVE. The positive allele was from LM001 (Table 3). The remaining five QTL detected in a single or two environments explained between 4.56 and $18.59 \%$ of the PVE.

Furthermore, five QTL for KW explained 13.91 to 39.01\% of the PVE. QKW.sicau-AM-4B, a major QTL, detected in all the six environments and also the BLUP data. This locus could explain 13.91 to $39.01 \%$ of the PVE, and the positive allele was contributed by Ailanmai (Table 3). The other four QTL were detected in less than three environments, and they explained between 22.96 and 29.82\% of the PVE (Table 3). Furthermore, twenty-eight QTL 

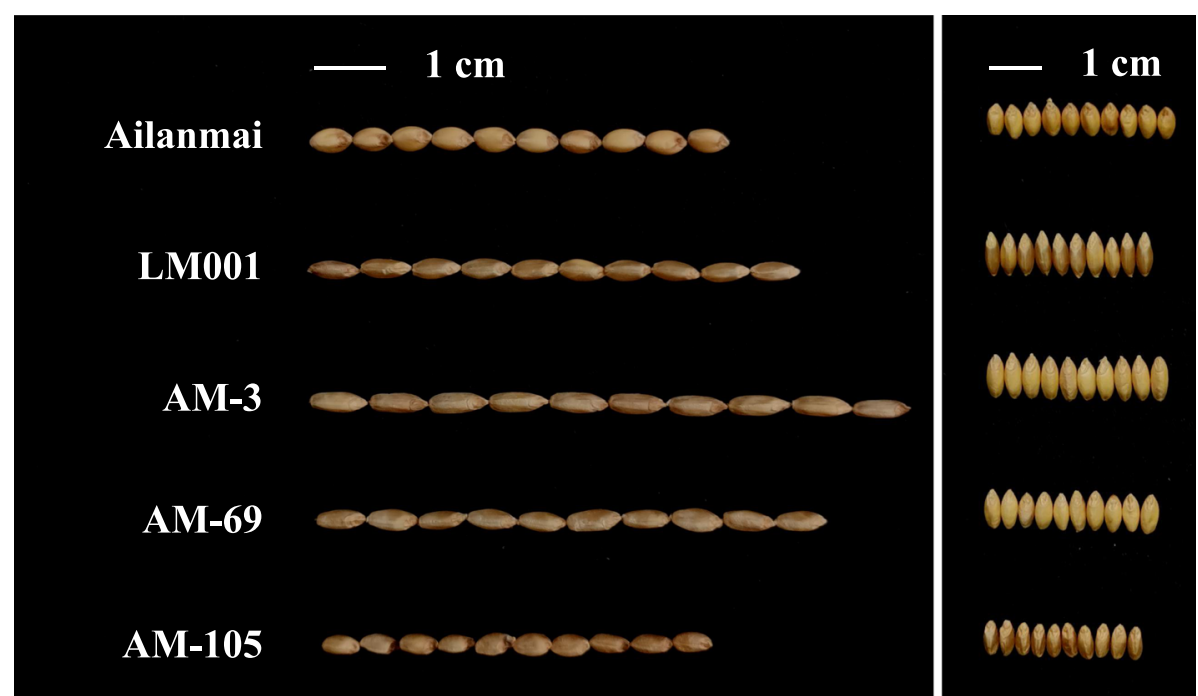

Fig. 1 Phenotypes of the parents Ailanmai, LM001 and partial RILs. Comparison of kernel length and width among parents Ailanmai, LM001 and partial RIL (i.e. AM-3, AM-69 and AM-105). The white bar represents the scale $=1 \mathrm{~cm}$

were detected using QE interaction analysis (Table S4). QKL.sicau-AM-3B controlling KL and QKW.sicau- $A M-4 B$ controlling $\mathrm{KW}$ were simultaneously identified by multienvironmental and individual environmental analyses, further showing that they were major and stable QTL.

\section{Verification of the major QTL in different genetic backgrounds}

A KASP marker, $K A S P-A X-111112626$, tightly linked to QKL.sicau-AM-3B and one, KASP-AX-108974756, tightly linked to $Q K W$.sicau- $A M-4 B$ were developed to validate their effects in different genetic backgrounds (Table S3; Fig. 2).

According to the polymorphism of KASP-AX111112626, the lines were divided into two groups in the AM RIL population: lines with Ailanmai homozygous allele and lines with LM001 homozygous allele (excluding heterozygosis). The group with positive allele of QKL.sicau- $A M-3 B$ (from LM001) had significantly greater values than that with negative one (from Ailanmai) in

Table 1 Phenotype variance explained (PVE), standard deviation (STD) and the broad-sense heritability $\left(H^{2}\right)$ of kernel length $(K L)$ and width (KW) for the parents and AM RIL population in different environments

\begin{tabular}{|c|c|c|c|c|c|c|c|}
\hline \multirow[t]{2}{*}{ Trait } & \multirow[t]{2}{*}{ Environment } & \multicolumn{2}{|l|}{ Parents } & \multicolumn{4}{|c|}{ Ailanmai × LM001 (AM) } \\
\hline & & Ailanmai & LM001 & Range & Mean & STD & $H^{2}$ \\
\hline \multirow[t]{7}{*}{$\mathrm{KL}(\mathrm{mm})$} & $2017 C Z$ & $N$ & $N$ & $5.96-9.06$ & 8.11 & 0.56 & 0.79 \\
\hline & 2018CZ & $6.28^{* *}$ & 8.15 & $5.90-9.00$ & 8.14 & 0.58 & \\
\hline & $2019 C Z$ & $6.67^{* *}$ & 8.65 & $6.59-9.40$ & 8.46 & 0.52 & \\
\hline & $2020 C Z$ & $6.28^{* *}$ & 8.35 & $6.51-9.43$ & 8.48 & 0.54 & \\
\hline & 2020WJ & $6.56^{* *}$ & 8.49 & $5.86-9.16$ & 8.25 & 0.60 & \\
\hline & 2020YA & $6.70^{* *}$ & 8.52 & $7.18-9.11$ & 8.46 & 0.45 & \\
\hline & BLUP & $6.58^{* *}$ & 8.39 & $6.47-9.04$ & 8.31 & 0.46 & \\
\hline \multirow[t]{7}{*}{ KW (mm) } & $2017 C Z$ & $N$ & $\mathrm{~N}$ & $2.89-3.72$ & 3.43 & 0.19 & 0.72 \\
\hline & $2018 C Z$ & $3.90^{* *}$ & 3.34 & $3.16-3.82$ & 3.54 & 0.15 & \\
\hline & 2019CZ & $3.92^{* *}$ & 3.42 & $3.21-4.16$ & 3.64 & 0.20 & \\
\hline & $2020 C Z$ & $3.96^{* *}$ & 3.35 & $2.74-3.95$ & 3.49 & 0.24 & \\
\hline & 2020WJ & $3.91^{* *}$ & 3.33 & $3.19-4.14$ & 3.58 & 0.18 & \\
\hline & 2020YA & $3.81^{* *}$ & 3.37 & $2.92-3.87$ & 3.46 & 0.20 & \\
\hline & BLUP & $3.82^{* *}$ & 3.38 & $3.26-3.80$ & 3.52 & 0.11 & \\
\hline
\end{tabular}

KL kernel length, KW kernel width, CZ Chongzhou, WJ Wenjiang, YA Ya'an, STD standard deviation, $H^{2}$ the broad-sense heritability, BLUP phenotype values based on BLUP, $N$ the data was missing; ${ }^{* *}$ Significance at the 0.01 probability level; ${ }^{*}$ Significance at the 0.05 probability level 
Table 2 Correlation coefficients of $\mathrm{KL}$ and $\mathrm{KW}$ in different environments

\begin{tabular}{|c|c|c|c|c|c|c|c|}
\hline Trait & Environment & $2017 C Z$ & $2018 C Z$ & $2019 C Z$ & $2020 C Z$ & 2020WJ & 2020YA \\
\hline \multirow[t]{6}{*}{$\mathrm{KL}$} & $2017 C Z$ & 1 & & & & & \\
\hline & $2018 C Z$ & $0.72^{* *}$ & 1 & & & & \\
\hline & $2019 C Z$ & $0.67^{* *}$ & $0.82^{* *}$ & 1 & & & \\
\hline & $2020 C Z$ & $0.62^{* *}$ & $0.70^{* *}$ & $0.77^{* *}$ & 1 & & \\
\hline & 2020WJ & $0.65^{* *}$ & $0.71^{* *}$ & $0.81^{* *}$ & $0.69^{* *}$ & 1 & \\
\hline & 2020YA & $0.76^{* *}$ & $0.79^{* *}$ & $0.80^{* *}$ & $0.72^{* *}$ & $0.75^{* *}$ & 1 \\
\hline \multirow[t]{6}{*}{ KW } & $2017 C Z$ & 1 & & & & & \\
\hline & $2018 C Z$ & $0.33^{* *}$ & 1 & & & & \\
\hline & $2019 C Z$ & $0.45^{* *}$ & 0.12 & 1 & & & \\
\hline & $2020 C Z$ & $0.43^{* *}$ & $0.29^{* *}$ & $0.36^{* *}$ & 1 & & \\
\hline & 2020WJ & 0.26 & $0.33^{* *}$ & $0.33^{* *}$ & $0.45^{* *}$ & 1 & \\
\hline & 2020YA & $0.43^{* *}$ & $0.29^{* *}$ & $0.36^{* *}$ & $0.37^{* *}$ & $0.29^{* *}$ & 1 \\
\hline
\end{tabular}

KL kernel length, KW kernel width, $C Z$ Chongzhou, WJ Wenjiang, YA Ya'an; ${ }^{* *}$ Significance at the 0.01 probability level; ${ }^{*}$ Significance at the 0.05 probability level

Table 3 Quantitative trait loci (QTL) mapping for kernel length (KL) and kernel width (KW)

\begin{tabular}{|c|c|c|c|c|c|c|c|c|c|}
\hline Trait & QTL & Environment & Chromosome & Left Marker & Right Marker & LOD & PVE (\%) & Add & $\mathrm{Cl}$ \\
\hline \multirow[t]{11}{*}{$\mathrm{KL}$} & QKL.sicau-AM-6A & $2019 C Z$ & $6 \mathrm{~A}$ & $A X-109843320$ & $A X-108755285$ & 2.55 & 4.56 & -0.12 & $10.5-21.5$ \\
\hline & QKL.sicau-AM-2B & $2018 C Z$ & $2 B$ & $A X-108730087$ & $A X-110447950$ & 3.06 & 5.38 & -0.20 & $21.5-37.5$ \\
\hline & \multirow[t]{6}{*}{ QKL.sicau-AM-3B } & $2018 C Z$ & $3 B$ & $A X-1111112626$ & $A X-110375013$ & 4.22 & 17.57 & -0.24 & $1.5-7.5$ \\
\hline & & $2019 C Z$ & & $A X-1111112626$ & $A X-110375013$ & 15.84 & 44.28 & -0.35 & $4.5-7.5$ \\
\hline & & $2020 C Z$ & & $A X-1111112626$ & $A X-110375013$ & 12.45 & 37.50 & -0.35 & $3.5-7.5$ \\
\hline & & 2020WJ & & $A X-1111112626$ & $A X-110375013$ & 7.69 & 27.70 & -0.32 & $2.5-7.5$ \\
\hline & & 2020YA & & $A X-1111112626$ & $A X-110375013$ & 10.03 & 32.55 & -0.26 & $2.5-7.5$ \\
\hline & & BLUP & & $A X-1111112626$ & $A X-110375013$ & 11.58 & 34.55 & -0.29 & $2.5-7.5$ \\
\hline & QKL.sicau-AM-3B.1 & $2017 C Z$ & $3 B$ & $A X-108914590$ & $A X-108727907$ & 3.90 & 13.57 & -0.21 & $10.5-13.5$ \\
\hline & QKL.sicau-AM-4B & BLUP & $4 B$ & $A X-109410422$ & $A X-94486277$ & 3.62 & 15.68 & 0.03 & $81.5-83.0$ \\
\hline & QKL.sicau-AM-6B & $2017 C Z$ & $6 \mathrm{~B}$ & $A X-109336882$ & $A X-110062048$ & 3.37 & 18.59 & 0.19 & $127.5-130.5$ \\
\hline \multirow[t]{11}{*}{ KW } & QKW.sicau-AM-2A & BLUP & $2 \mathrm{~A}$ & AX-108949998 & AX-109355803 & 3.38 & 22.96 & 0.03 & $134.5-140.5$ \\
\hline & QKW.sicau-AM-7A & BLUP & $7 \mathrm{~A}$ & $A X-108885515$ & $A X-109302546$ & 2.86 & 26.22 & -0.03 & $91.5-97.5$ \\
\hline & QKW.sicau-AM-1B & BLUP & $1 \mathrm{~B}$ & $A X-111062860$ & $A X-110067443$ & 4.98 & 29.08 & 0.04 & $79.5-81.5$ \\
\hline & QKW.sicau-AM-2B & 2019CZ & $2 \mathrm{~B}$ & $A X-109897880$ & $A X-110598098$ & 2.96 & 29.82 & 0.06 & $11.5-13.5$ \\
\hline & \multirow[t]{7}{*}{ QKW.sicau-AM-4B } & $2017 C Z$ & $4 \mathrm{~B}$ & $A X-108974756$ & $A X-111451315$ & 7.77 & 25.17 & 0.10 & $12.5-14.5$ \\
\hline & & $2018 C Z$ & & $A X-108974756$ & $A X-111451315$ & 10.10 & 38.44 & 0.09 & $12.5-14.5$ \\
\hline & & $2019 C Z$ & & $A X-108955591$ & $A X-110915030$ & 3.81 & 13.91 & 0.07 & $14.5-15.5$ \\
\hline & & $2020 C Z$ & & $A X-108955591$ & $A X-110915030$ & 8.91 & 29.97 & 0.13 & $14.5-15.5$ \\
\hline & & 2020WJ & & $A X-108955591$ & $A X-110915030$ & 5.98 & 23.31 & 0.08 & $14.5-15.5$ \\
\hline & & 2020YA & & $A X-108955591$ & $A X-110915030$ & 5.58 & 24.02 & 0.09 & $14.5-15.5$ \\
\hline & & BLUP & & $A X-108955591$ & $A X-110915030$ & 18.17 & 39.01 & 0.08 & $14.5-15.5$ \\
\hline
\end{tabular}

KL kernel length, KW kernel width, CZ Chongzhou, WJ Wenjiang, YA Ya'an, LOD logarithmic odds, PVE phenotype variation values. Add: additive effect of a QTL, positive values indicate that alleles from Ailanmai are increasing the trait scores, and negative values indicate that alleles from LM001 are increasing the trait scores. Cl: confidence interval of the QTL 


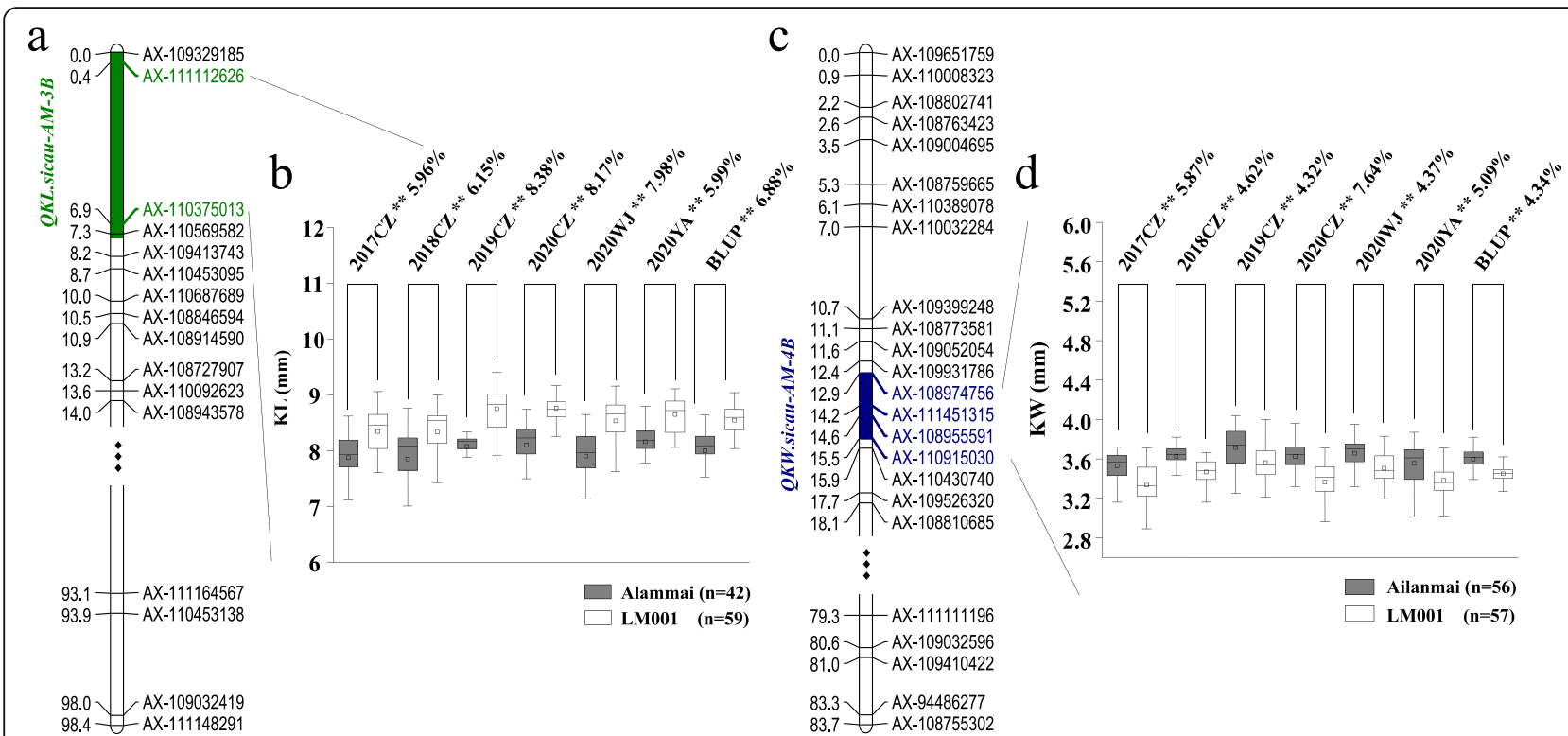

Fig. 2 Major QTL QKL.sicau-AM-3B and QKW.sicau-AM-4B and their effects. (a): Genetic map of chromosome 3B; the green area was the interval of QKL.sicau-AM-3B; (b): effect of QKL.sicau-AM-3B shown as box plots calculated after grouping the AM population into two classes based on the flanking markers. Ailanmai and LM001 indicate the lines with and without positive alleles of QKL.sicau-AM-3B; (c): genetic map of chromosome 4B; The blue area was the interval of QKW.sicau-AM-4B; (d): effect of QKW.sicau-AM-4B shown as box plots calculated after grouping the AM population into two classes based on the flanking markers. CZ: Chongzhou; WJ: Wenjiang; YA: Ya'an; ${ }^{* *}$ significance at the 0.01 probability level, ${ }^{*}$ significance at the 0.05 probability level. Differences between the two groups were labeled below the environmental names and BLUP

each environment and BLUP data set $(P<0.05$; Fig. 2a and b). Likewise, the lines from CWL population were divided into two groups. The group with positive allele of QKL.sicau-AM-3B had 3.29\% higher values than that with negative one $(P<0.05$; Fig. $3 \mathrm{~d})$. In MP population, the lines with positive allele had $11.46 \%$ higher values than those with negative one, indicating that QKL.sicau$A M-3 B$ is indeed a major QTL controlling KL (Fig. 3a).
Additionally, according to the polymorphism of KASP-AX-108974756, the lines from AM population were also divided into two groups. The group with positive allele of $Q K W$.sicau-AM- $4 B$ had significantly higher values than that with negative one in six environments and BLUP data set $(P<0.05$; Fig. $2 \mathrm{c}$, d). In $C W C$ population, the group with positive allele of QKW.sicau-AM- $4 B$ had significantly $0.78 \%$ greater
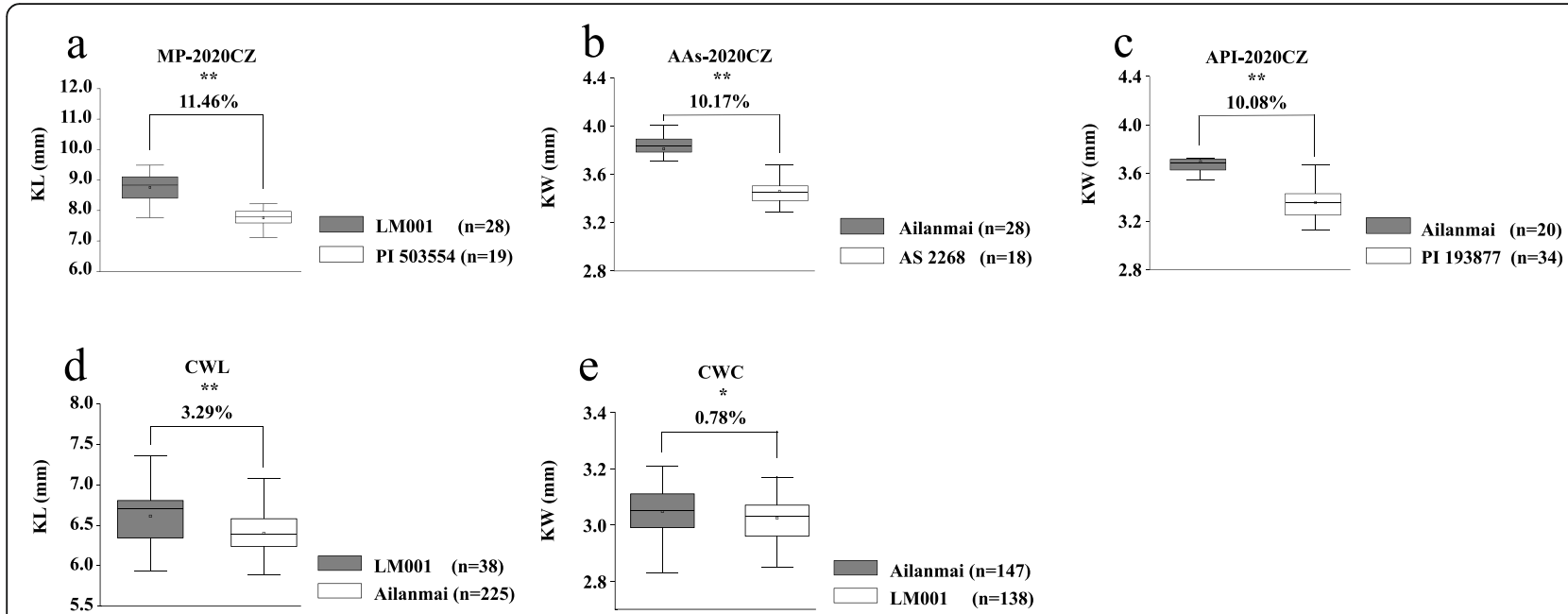

Fig. 3 Verification of $Q K L$.sicau-AM-3B and QKW.sicau-AM-4B in two and three different genetic background populations, respectively. (a), (d): Effects of QKL.sicau-AM-3B in the MP and the CWL validation populations, respectively; (b), (c), and (e): effects of QKW.sicau-AM-4B in the three populations (i.e. AAs, API and CWC). CZ: Chongzhou; ${ }^{* *}$ significance at the 0.01 probability level, ${ }^{*}$ significance at the 0.05 probability level 
values than that with negative one $(P<0.05$; Fig. $3 e)$. In AAs and API populations, the group with positive allele from Ailanmai had significantly greater values than that without this allele, and the differences between the two groups were 10.17 and $10.08 \%$, respectively, with an average of $10.13 \%$ in two validation populations, indicating that $Q K W$.sicau- $A M-4 B$ is also a major QTL controlling KW (Fig. 3b, c).

\section{Effects of $Q K L$.Sicau-AM-3B and QKW.Sicau-AM-4B on TKW in $A M$ population}

In AM population, the positive alleles of QKL.sicau-AM$3 B$ and $Q K W$.sicau-AM-4B were from LM001 and Ailanmai, respectively (Table 3). The effects of QKL.sicau$A M-3 B$ and $Q K W$.sicau- $A M-4 B$ on TKW were further analyzed (Fig. 5). Compared with those without any of the alleles increasing $\mathrm{KL}$ and $\mathrm{KW}$, lines possessed the positive allele of QKL.sicau-AM-3B but not that of $Q K W$. sicau- $A M-4 B$ increased TKW by $1.65 \%$; lines possessed that of $Q K W$.sicau-AM- $4 B$ but not that of QKL.sicau- $A M-3 B$ increased TKW by $1.49 \%$; and those with the combination of positive alleles of both QKL.sicau$A M-3 B$ and $Q K W$.sicau- $A M-4 B$ significantly increased TKW by up to $6.01 \%(P<0.01)$. Besides, lines with the combination of positive alleles of QKL.sicau-AM-3B and QKW.sicau- $A M-4 B$ significantly increased TKW by 4.29 and $4.46 \%(P<0.05)$, respectively, compared to those with the positive allele of the QKL.sicau- $A M-3 B$ only or the QKW.sicau-AM-4B only (Fig. 5). In addition, the binary linear regression analysis on TKW showed that the path coefficient of $\mathrm{KL}$ was 0.43 , and that of $\mathrm{KW}$ was 0.02 , indicating that $\mathrm{KL}$ contributes more to TKW than KW (Table S5).

\section{Discussion}

\section{Relationship between kernel size and other agronomic} traits

In this study, we evaluated the correlation coefficients between kernel size and other agronomic traits (Fig. S2). Positive and significant correlations were observed between KL, KW, and TKW $(P<0.05$; Fig. S2d, l). The result indicated that the selection of larger kernels might lead to indirect selection of heavier kernels [29]. Kernel size, like KL and KW, greatly influences TKW. For example, Cui et al. found that compared with other kernel traits, KW has the largest effect on TKW [30]. Liu et al. also reported that TKW was mainly affected by KW [31]. In the current study, KL likely contributed more to TKW than KW (Fig. 5; Table S5), suggesting that increasing KL through utilization of positive allele of QKL.sicau-AM-3B may be more effective in increasing TKW than KW contributed by positive allele of QKW.sicau- $A M-4 B$ at tetraploid level. As expected, KL and KW were positively correlated with UIL (Fig. S2c, k). A longer UIL contributed to ventilation, light transmittance, and lower relative humidity of spikes, thus reducing the possibility of occurrence of diseases and insect pests such as scab, which was conducive to dry matter accumulation and affects kernel size [32]. KL and FLL showed significant positive correlation, and KW was positively correlated with FLW $(P<0.05$; Fig. S2g, o). Theoretically, FLL and FLW determined the flag leaf area that was proportional to whether it had a strong assimilation tissue, vascular bundle area and these factors determined the kernel filling intensity of wheat, which was closely correlated with kernel size [33]. Furthermore, the results indicated that larger flag leaves increased yield by providing more photosynthetic nutrient to kernel [34]. The above conclusions provided a scientific basis for evaluating complex relationships among wheat yield components, which will be helpful in understanding increase of wheat yield.

\section{Stable and novel QTL controlling KL and KW}

We compared the major QTL identified in this study with those detected in previous studies through aligning physical positions of their closest markers (Table S6).

QKL.sicau-AM-3B was located between 675.6 and $695.4 \mathrm{Mb}$ in the deletion bin 3BL7-0.63-1.00 on chromosome arm 3BL in wild emmer (Fig. 4a, c), which was different from previously reported KL-related QTL (Table S6). For example, QGl-3B.1 was detected on chromosome arm 3BS at 52.1-53.2 Mb [35]. And QGl.ccsu-3B.1 was flanked by marker Xgwm376 (38.9 $\mathrm{Mb})$ [36]. Two QTL, QKL.ndsu.3B and QKL.ndsu.3B.1, were located at $211.7-216.3 \mathrm{Mb}$ and $233.9-244.6 \mathrm{Mb}$, respectively [37]. And $q K L .3 B$ was identified on chromosome arm 3BS with the closest marker Xgwm429 (20.5 $\mathrm{Mb}$ ) [38]. Thus, these results indicated QKL.sicau-AM$3 B$ may be a novel QTL controlling KL detected in the present study.

For KW, QKW.sicau-AM- $4 B$ was located between 444.2 and $474.0 \mathrm{Mb}$ in the deletion bin $4 \mathrm{BL} 1-0.71$ and 4BL1-0.71-0.86 on chromosome arm 4BL in wild emmer (Fig. 4e, g). Comparison of physical positions of QKW.sicau-AM-4B with those reported previously suggested that they were not overlapped (Table S6). For example, there were five QTL, QKw.ncl-4B.1 [39], QGw$4 B .1$ [35], Size width 2011 [14], QKw4B.1-7 [40], QKw$4 B .2$ [41], and $k w-W Y-4 B-1.2$ [30], associated with $\mathrm{KW}$ being detected on chromosome arm 4BS. $q K W-4 B$ was identified on chromosome arm 4BL with the closest marker Xwmc657 (610.5 Mb) [38], and $q K W 4 B-1$ was detected on chromosome arm 4BL at $610.1-649.1 \mathrm{Mb}$ [8]. These results showed that QKW.sicau-AM- $4 B$ is probably a novel QTL controlling KW in wheat. 


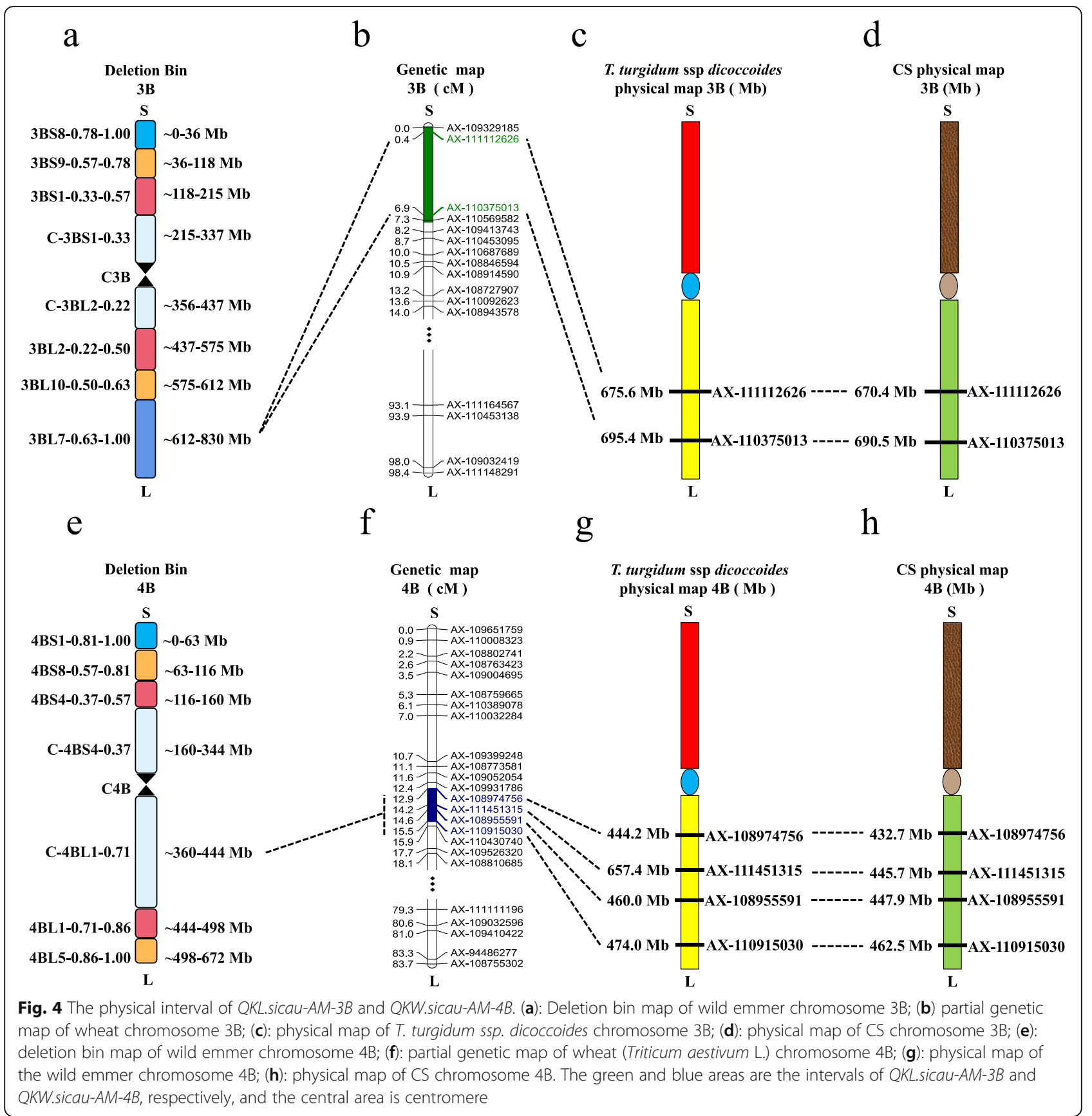

Predictive genes in the intervals where major QTL were located

QKL.sicau-AM-3B was located between 675.6 and 695.4 $\mathrm{Mb}$ on wild emmer 3BL and between 670.4 and 690.5 $\mathrm{Mb}$ on $\mathrm{CS} 3 \mathrm{BL}$ by anchoring flanking markers $A X$ 111112626 and $A X-110375013$ of QKL.sicau-AM-3B (Fig. 4a, b, c, and d). There were twenty-nine shared predicated genes (Table S7). Expression analyses showed that twenty-four genes can be expressed in kernel (Fig. S3a). Similarly, for $Q K W$.sicau-AM-4B, it was mapped between 444.2 and $474.0 \mathrm{Mb}$ on chromosome arm $4 \mathrm{BL}$ of wild emmer and 432.7 and $462.5 \mathrm{Mb}$ on chromosome arm $4 \mathrm{BL}$ of $\mathrm{CS}$ by anchoring its flanking markers $A X$ 108974756 and $A X-110915030$ (Fig. 4e, f, g and h). There were forty shared predicated genes (Table S7). Expression analyses showed that thirty-four genes can be expressed in kernel (Fig. S3b).

Of these sixty-nine genes, four genes were involved in kernel development. For example, TRIDC3BG062390 encoded fructose-bisphosphate aldolase (FBA) and it had 


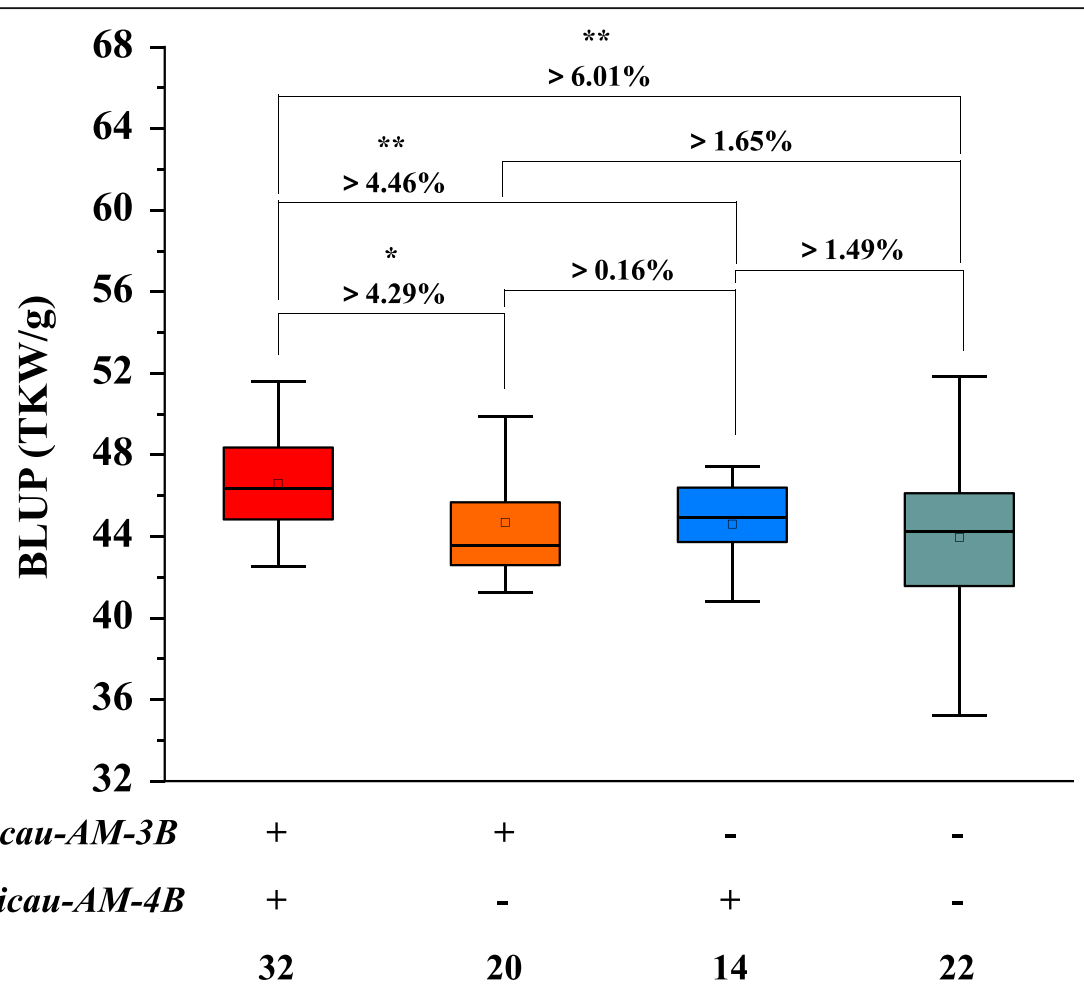

Fig. 5 The effects of different combinations of QKL.sicau-AM-3B and QKW.sicau-AM-4B on increasing 1000-kernel weight (TKW) in the AM population. ' + ' and '-' represent lines with and without the positive allele of the corresponding QTL based on the genotype of flanking markers, respectively. ${ }^{* *}$ Significance at the 0.01 probability level, ${ }^{*}$ significance at the 0.05 probability level

a higher expression in kernel than other genes (Fig. S3a). FBA is an important isozyme involved in plant metabolism, and it is directly involved in the fixation and distribution of photosynthate [42]. Cytosolic and plastidic FBAs were expressed in plant photosynthetic tissues [43]. FBA regulates kernel size development through affecting plant photosynthesis. In addition, there were two un-functional and annotated genes of wild emmer, TRIDC4BG037810 and TRIDC4BG037830. Nonetheless, they were highly expressed in kernel at different growth stages (Fig. S4a, b). Therefore, we identified annotations of their orthologs in CS [28]. TraesCS4B03G0584900 (TRIDC4BG037810) and TraesCS4B03G0585000 (TRIDC4BG037830) encoded Heat-shock protein (HSP; Table S7) and were also highly expressed in kernel (Fig. S4c, d). HSP was widely reported in graminaceous plant [44]. At high temperature, the role of HSP is to ensure the normal growth of kernel in wheat through providing protection to soluble starch synthase [45]. It was reported that HSP, as a molecular chaperone, aids in refolding soluble starch synthases denatured by heat and thus prevents them from aggregating, which was beneficial to starch synthesis of in kernel [46]. Thus, these genes related to kernel development may provide information for fine mapping and gene cloning of these identified major and novel QTL.

\section{Utilization of elite alleles for kernel size from wheat related species}

In the current study, two major, stably expressed, and novel QTL, QKL.sicau-AM-3B and QKW.sicau-AM-4B for kernel-related traits were identified from a wild emmer accession and a local landrace and validated in five populations with different genetic backgrounds. The combination of QKL.sicau-AM-3B and QKW.sicau-AM$4 B$ had the largest additive effect on TKW (Fig. 5). These results suggest that they have a great potential in wheat breeding. Previous studies showed that pyramiding of choiceness genes was an effective method to improve a given trait [47]. In this study, we found some transgressive segregations in AM RIL. For example, AM-3 has longer and wider kernels than both parents (Fig. 1). Interestingly, AM-3 carries the positive alleles of both QKL.sicau-AM-3B and QKW.sicau-AM-4B, implying the possibility of pyramiding these two positive alleles from wheat related species in wheat breeding.

\section{Conclusions}

Two major and novel QTL, QKL.sicau-AM-3B and QKW.sicau-AM-4B, were identified in AM RIL population. Both of them were successfully verified in their corresponding validation populations with newly developed KASP makers. Some genes involved in regulation of 
kernel growth and development were detected in the intervals where major $\mathrm{KL}$ and $\mathrm{KW}$ QTL were located. Significant correlations between kernel size and other agronomic traits were detected and discussed. KASP markers tightly linked the two major QTL could contribute greatly to subsequent fine mapping. This study indicated that wheat related species have great potentials for wheat yield improvement.

\section{Abbreviations}

QTL: Quantitative trait loci; KL: Kernel length; KW: Kernel width; TKW: 1000kernel weight; SL: Spike length; ETN: Effective tiller number; UIL: Length of uppermost internode; GNS: Grain number per spike; FLL: Flag leaf length; FLW: Flag leaf width; AM: Ailanmai $\times$ LM001; RILs: Recombinant inbred lines; PVE: Phenotypic variance explained; KASP: Kompetitive allele-specific PCR: SNP: Single-nucleotide polymorphism; MP: LM001 × PI 503554; AAs: Ailanmai $\times$ AS 2268; API: Ailanmai × PI 193877; NPGS: The U.S. National Plant Germplasm System; CWL: Chinese wheat landraces; CWC: Chinese wheat cultivars; CZ: Chongzhou; WJ: Wenjiang; YA: Ya'an; BLUP: Best linear unbiased prediction; $H^{2}$ : Broad-sense heritability; LOD: Logarithm of odds; QE: QTL $\times$ Environment; CS: Chinese Spring; STD: Standard deviation; FBA: Fructosebisphosphate aldolase; HSP: Heat-shock protein

\section{Supplementary Information}

The online version contains supplementary material available at https://doi. org/10.1186/s12864-021-08024-z.

\section{Additional file 1: Table S1. Information of CWL and CWC natural populations assessed in this study.}

Additional file 2: Table S2. Environmental information of examined agronomic traits.

Additional file 3: Table S3. Details of KASP primers used and the amplification reaction system and conditions in this study.

Additional file 4: Figure S1. Phenotypic distribution of kernel length $(\mathrm{KL})$ and width (KW) at different environments and BLUP. (a): Frequency distribution map of $\mathrm{KL} ;(\mathbf{b})$ : frequency distribution map of KW. Black and gray arrows represent the parents Ailanmai and LM001, respectively.

Additional file 5: Figure S2. Correlation analysis for kernel traits (KL and $\mathrm{KW}$ ) with (a) and (i): spike length (SL); (b) and (j): effective tiller number (ETN); (c) and (k): length of uppermost internode (UIL); (d) and (I): 1,000-kernel weight (TKW); (e) and (m): grain number per spike (GNS); $(\mathbf{f})$ : kernel width and length (KW \& KL); $(\mathbf{g})$ and $(\mathbf{n})$ : flag leaf length (FLL); (h) and (o): flag leaf width (FLW), respectively. ${ }^{* *}$ Significance at the 0.01 probability level, ${ }^{*}$ significance at the 0.05 probability level.

Additional file 6: Table S4. Quantitative trait loci (QTL) detected in the QTL $\times$ environment interaction module (QE).

Additional file 7: Table S5. Results of the linear regression analysis on TKW.

Additional file 8: Table S6. Comparison of QKL.sicau-AM-3B and QKW.sicau-AM-4B with previous reported quantitative trait loci (QTL) or marker-trait associations (MTAs) for kernel length $(\mathrm{KL})$ and kernel width $(\mathrm{KW})$, respectively.

Additional file 9: Table S7. Predicated genes in the interval of QKL.sicau-AM-3B and OKW.sicau-AM-4B.

Additional file 10: Figure S3. Expression analysis of predictive genes in the interval of QKL.sicau-AM-3B (a) and QKW.sicau-AM-4B (b) in kernel.

Additional file 11: Figure S4. The expression of TRIDC4BG037810 (a) and TRIDC4BG037830 (b), and TraesCS4B03G0584900 (c) and TraesCS4B03G0585000 (d) in different growth stages of wild emmer and $\mathrm{CS}$, respectively.

\section{Authors' contributions}

JGZ and LC conducted the entire study and drafted this manuscript. JNY did phenotype measurement. HPT, YM, Jun M, and YTG did field work. QTJ, and YXL helped with data analysis. GYC, JRW, PFQ, and AH did QTL analysis and manuscript revision. YMW revised manuscript. YLZ discussed results and revised the manuscript. $X J L$ guided the study and revised the manuscript. Jian $M$ designed the experiments, guided the entire study, participated in data analysis, wrote and extensively revised this manuscript. All authors participated in the research and approved the final manuscript.

\section{Funding}

This work is supported by the National Natural Science Foundation of China (31970243 and 31971937), the International Science and Technology Cooperation and Exchanges Program of Science and Technology Department of Sichuan Province (2021YFH0083), the Applied Basic Research Programs of Science and Technology Department of Sichuan Province (2020YJ0140 and 2021YJ0503), and the Basic Research Project of Science and Technology Plan of Guizhou Province (ZK [2021] General 131). We thank the anonymous referees for reading and revising this manuscript.

\section{Availability of data and materials}

The data that support the findings of this study are available in 'figshare' with the identifiers data DOls, including dataset 1 (data of KL and KW of AM RILs in six environments, https://doi.org/10.6084/m9.figshare.14813094.v1), dataset 2 (the phenotypes and genotypes of MP, AAs and API populations, https://doi. org/10.6084/m9.figshare.14813109.v2). Remaining data generated or analyzed during this study are included in this published article and its Additional files.

\section{Declarations}

\section{Ethics approval and consent to participate}

The wheat accessions (Ailanmai, LM001, AS 2268 and PI 503554, PI 193877) materials used in the experiment were supplied by Triticeae Research Institute of Sichuan Agricultural University and The U.S. National Plant Germplasm System (NPGS), respectively. These plant materials are widely used all over the world and no permits are required for the collection of plant samples. The plant materials are maintained in accordance with the institutional guidelines of Triticeae Research Institute of Sichuan Agricultural University, China. Experimental research and field studies on plants, including the collection of plant material, complied with relevant institutional, national, and international guidelines and legislation. Furthermore, this article did not contain any studies with human participants or animals and did not involve any endangered or protected species. All experiments and data analyses were conducted in Sichuan. All authors contributed to the study and approved the final version for submission. The manuscript has not been submitted to any other journal.

\section{Consent for publication}

Not applicable.

\section{Competing interests}

All authors declare that there is no conflict of interest.

\section{Author details}

${ }^{1}$ State Key Laboratory of Crop Gene Exploration and Utilization in Southwest China, Triticeae Research Institute, Sichuan Agricultural University, Chengdu 611130, China. ${ }^{2}$ College of Agronomy and Biotechnology, China Agricultural University, Beijing 100193, China. ${ }^{3}$ Biotechnology and Genetic Engineering Discipline, Khulna University, Khulna 9208, Bangladesh.

Received: 6 April 2021 Accepted: 13 September 2021 Published online: 30 September 2021

\section{References}

1. Wang DW, Li F, Cao SH, Zhang KP. Genomic and functional genomics analyses of gluten proteins and prospect for simultaneous improvement of end-use and health-related traits in wheat. Theor Appl Genet. 2020;133(5): $1521-39$.

2. Zhao YL, Yang X, Zhou GH, Zhang T. Engineering plant virus resistance: from RNA silencing to genome editing strategies. Plant Biotechnol J. 2020; 18(2):328-36. 
3. Fan XL, Cui F, Ji J, Zhang W, Zhao XQ, Liu JJ, et al. Dissection of pleiotropic QTL regions controlling wheat spike characteristics under different nitrogen treatments using traditional and conditional QTL mapping. Front Plant Sci. 2019;10:187.

4. Desiderio F, Zarei L, Licciardello S, Cheghamirza K, Farshadfar E, Virzi N, et al. Genomic regions from an Iranian landrace increase kernel size in durum wheat. Front Plant Sci. 2019;10:448.

5. Yan XF, Zhao L, Ren Y, Dong ZD, Cui DQ, Chen F. Genome-wide association study revealed that the TaGW8 gene was associated with kernel size in Chinese bread wheat. Sci Rep. 2019;9(1):2702.

6. Li MX, Wang ZL, Liang ZY, Shen WN, Sun FL, Xi YJ, et al. Quantitative trait loci analysis for kernel-related characteristics in common wheat (Triticum aestivum L.). Crop Sci. 2015:55:1-9.

7. Prashant R, Kadoo N, Desale C, Kore P, Dhaliwal HS, Chhuneja P, et al. Kernel morphometric traits in hexaploid wheat (Triticum aestivum L.) are modulated by intricate QTL $\times$ QTL and genotype $\times$ environment interactions. J Cereal Sci. 2012;56(2):432-9.

8. Xin F, Zhu T, Wei SW, Han YC, Zhao Y, Zhang DZ, et al. QTL mapping of kernel traits and validation of a major QTL for kernel length-width ratio using SNP and bulked segregant analysis in wheat. Sci Rep. 2020;10(1):1-12.

9. Cheng $X J$, Xin MM, Xu RB, Chen ZY, Cai WL, Chai $L L$, et al. A single amino acid substitution in STK__GSK3 kinase conferring semispherical grains and its implications for the origin of Triticum sphaerococcum. Plant Cell. 2020; 32(4):923-34.

10. Chen $Y$, Yan $Y$, Wu TT, Zhang GL, Yin HR, Chen W, et al. Cloning of wheat keto-acyl thiolase $2 B$ reveals a role of jasmonic acid in grain weight determination. Nat Commun. 2020;11(1):1-11.

11. Lin $Y$, Chen GD, Hu HY, Yang XL, Zhang ZL, Jiang XJ, et al. Phenotypic and genetic variation in phosphorus-deficiency-tolerance traits in Chinese wheat landraces. BMC Plant Biol. 2020;20(1):1-9.

12. Li XJ, Jiang XL, Chen XD, Song J, Ren CC, Xiao YJ, et al. Molecular cytogenetic identification of a novel wheat-Agropyron elongatum chromosome translocation line with powdery mildew resistance. PLoS One. 2017;12(9):e0184462.

13. Assaf D, Hale I, Batsheva B-Z, Moran N, Raz A. QTLs for uniform grain dimensions and germination selected during wheat domestication are colocated on chromosome 4B. Theor Appl Genet. 2016;129:1303-15.

14. Russo MA, Ficco DBM, Laido G, Marone D, Papa R, Blanco A, et al. A dense durum wheat $\times T$. dicoccum linkage map based on SNP markers for the study of seed morphology. Mol Breed. 2014;34(4):1579-97.

15. Okamoto Y, Takumi S. Pleiotropic effects of the elongated glume gene $P 1$ on grain and spikelet shape-related traits in tetraploid wheat. Euphytica. 2013;194(2):207-18.

16. Avni R, Oren L, Shabtay G, Assili S, Distelfeld A. Genome based meta-QTL analysis of grain weight in tetraploid wheat identifies rare alleles of GRF4 associated with larger grains. Genes. 2018;9(12):636.

17. Zhou Y, Zhao XB, Li YW, Xu J, Bi AY, Kang LP, et al. Triticum population sequencing provides insights into wheat adaptation. Nat Genet. 2020;52(12): 1412-22.

18. Mo ZQ, Zhu J, Wei JT, Zhou JG, Xu Q, Tang HP, et al. The wheat 55K SNPbased exploration of loci for spikelet number per spike from a tetraploid wheat (Triticum turgidum L.) recombinant inbred line population derived from a Chinese landrace 'Ailanmai' and a wild emmer accession. bioRxiv. 2020. https://doi.org/10.1101/2020.10.21.348227.

19. Liu YX, Lin Y, Gao S, Li ZY, Ma J, Deng M, et al. A genome-wide association study of 23 agronomic traits in Chinese wheat landraces. Plant J. 2017;91(5): $861-73$.

20. Gao YT, Xu XR, Jin JJ, Duan SN, Zhen WC, Xie CJ, et al. Dissecting the genetic basis of grain morphology traits in Chinese wheat by genome wide association study. Euphytica. 2021;217(4):56.

21. Ma J, Zhang H, Li SQ, Zou Y, Li T, Liu JJ, et al. Identification of quantitative trait loci for kernel traits in a wheat cultivar Chuannong16. BMC Genet. 2019;20(1):77.

22. Lu P, Qin JX, Wang GX, Wang LL, Wang ZZ, Wu QH, et al. Comparative fine mapping of the wax 1 (W1) locus in hexaploid wheat. Theor Appl Genet. 2015;128(8):1595-603.

23. Smith SE, Kuehl R, Ray I, Hui R, Soleri D. Evaluation of simple methods for estimating broad-sense heritability in stands of randomly planted genotypes. Crop Sci. 1998;38(5):1125-9.

24. Churchill GA, Doerge RW. Empirical threshold values for quantitative trait mapping. Genetics. 1994;138(3):963-71.
25. Ma J, Ding PY, Liu JJ, Li T, Zou YY, Habib A, et al. Identification and validation of a major and stably expressed QTL for spikelet number per spike in bread wheat. Theor Appl Genet. 2019;132(11):3155-67.

26. Liu JJ, Luo W, Qin NN, Ding PY, Zhang H, Yang CC, et al. A 55 K SNP arraybased genetic map and its utilization in QTL mapping for productive tiller number in common wheat. Theor Appl Genet. 2018;131(11):2439-50.

27. Zhu T, Wang L, Rodriguez J, Deal K, Avni R, Distelfeld A, et al. Improved Genome Sequence of Wild Emmer Wheat Zavitan with the Aid of Optical Maps. G3. 2019;9(3):619-24.

28. Zhu T, Wang L, Rimbert H, Rodriguez JC, Deal KR, De Oliveira R, et al. Optical maps refine the bread wheat Triticum aestivum cv. Chinese Spring genome assembly. Plant J. 2021;107:303-14.

29. Ammiraju J, Dholakia B, Santra D, Singh H, Lagu M, Tamhankar S, et al. Identification of inter simple sequence repeat (ISSR) markers associated with seed size in wheat. Theor Appl Genet. 2001;102(5):726-32.

30. Cui F, Ding A, Li J, Zhao C, Li X, Feng D, et al. Wheat kernel dimensions: how do they contribute to kernel weight at an individual QTL level? J Genet. 2011;90(3):409-25.

31. Liu H, Zhang X, Xu Y, Ma F, Zhang J, Cao Y, et al. Identification and validation of quantitative trait loci for kernel traits in common wheat (Triticum aestivum L.). BMC Plant Biol. 2020;20(529):1-15.

32. Ma J, Min S, Puyang D, Wei L, Xiaohong Z, Congcong Y, et al. Genetic identification of QTL for neck length of spike in wheat. J Triticeae Crop. 2017;3:319-24 (in Chineses).

33. Yang T, Guo TC, Luo Y, Sun JH. Relationship between the anatomical structure and the grain formation of winter wheat varieties with different grain types. Acta Agron Sin. 1998;24(6):876-83 (in Chineses).

34. Liu KY, Xu H, Liu G, Guan PF, Zhou XY, Peng HR, et al. QTL mapping of flag leaf-related traits in wheat (Triticum aestivum L.). Theor Appl Genet. 2018; 131(4):839-49.

35. Zhang GZ. Construction of high density genetic map and QTL analysis for yield, spike and grain size traits in wheat. J Shandong Agr Univ. 2014; (in Chineses).

36. Tyagi S, Mir RR, Balyan HS, Gupta PK. Interval mapping and meta-QTL analysis of grain traits in common wheat ( Triticum aestivum L.). Euphytica. 2015;201(3):367-80.

37. Kumar A, Mantovani EE, Seetan R, Soltani A. M ES. Dissection of genetic factors underlying wheat kernel shape and size in an elite $x$ nonadapted cross using a high density SNP linkage map. Plant Genome-US. 2016;9(1):1-22.

38. Chen WG, Sun DZ, Yan X, Li RZ, Jing RL. QTL analysis of wheat kernel traits, and genetic effects of $q K W-6 A$ on kernel width. Euphytica. 2019;215:11.

39. Ramya P, Chaubal A, Kulkarni K, Gupta L, Kadoo N, Dhaliwal HS, et al. QTL mapping of 1000-kernel weight, kernel length, and kernel width in bread wheat (Triticum aestivum L.). J Appl Genet. 2010;51(4):421-9.

40. Li QF, Zhang Y, Liu TT, Wang FF, Liu K, Chen JS, et al. Genetic analysis of kernel weight and kernel size in wheat (Triticum aestivum L.) using unconditional and conditional QTL mapping. Mol Breed. 2015;35(10):194.

41. Cui F, Fan XL, Chen M, Zhang N, Zhao CH, Zhang W, et al. QTL detection for wheat kernel size and quality and the responses of these traits to low nitrogen stress. Theor Appl Genet. 2016;129(3):469-84.

42. Martin W, Mustafa AZ, Schnarrenberger HC. Higher-plant chloroplast and cytosolic fructose-1,6-bisphophosphatase isoenzymes: origins via duplication rather than prokaryote-eukaryote divergence. Plant Mol Biol. 1996;32:485-91.

43. Plaxton WC. The organization and regulation of plant glycolysis. Plant Mol Biol. 1996:47:185-214

44. Chen JF, Gao T, Wan SQ, Zhang YH, Yang JK, Yu YB, et al. Genome-wide identification, classification and expression analysis of the HSP gene superfamily in tea plant (Camellia sinensis). Int J Mol Sci. 2018;19(9):2633.

45. Prakash P, Sharma-Natu P, Ghildiyal MC. High temperature effect on starch synthase activity in relation to grain growth in wheat cultivars. Indian J Plant Physiol. 2003:8:390-8.

46. Iba K. Acclimative response to temperature stress in higher plants: approaches of gene engineering for temperature tolerance. Annu Rev Plant Biol. 2002;53:225-45.

47. Fan XL, Cui F, Zhao CH, Zhang W, Yang LJ, Zhao XQ, et al. QTLs for flag leaf size and their influence on yield-related traits in wheat (Triticum aestivum L.) Mol Breed. 2015;35(24):1-16.

\section{Publisher's Note}

Springer Nature remains neutral with regard to jurisdictional claims in published maps and institutional affiliations. 